\title{
Oviposition site attraction of Aedes albopictus to sites with conspecific and heterospecific larvae during an ongoing invasion in Medellín, Colombia
}

Talya Shragai ${ }^{1}$, Laura Harrington ${ }^{1 *} \mathbb{0}$, Catalina Alfonso-Parra ${ }^{2,3}$ and Frank Avila ${ }^{3}$

\begin{abstract}
Background: Aedes aegypti and Aedes albopictus are two globally invasive vectors with similar ecological niches. Encounters between them can result in either competitive exclusion or stable co-existence, but it is unclear what drives these variable outcomes. Larval competition in favor of Ae. albopictus is a main hypothesis for the competitive exclusion of Ae. aegypti observed in some regions. However, the role of oviposition preference in determining the degree of competitive larval interactions in the field is not well understood. In this study, we used a combination of mark-release-recapture methods with ovitraps in the open-field and a semi-field cage to test whether gravid Ae. albopictus seek oviposition sites in response to the presence, species, and density of either conspecific or heterospecific Ae. aegypti larvae in the aquatic habitat. We conducted our study in Medellín, Colombia, where Ae. aegypti is a long-term resident and Ae. albopictus is a recent invader.
\end{abstract}

Results: In the open-field and semi-field cage experiments, gravid Ae. albopictus showed strong preference for ovitraps with larvae over those without. They consistently preferred ovitraps with higher density of conspecific (Ae. albopictus) larvae and low density of heterospecific (Ae. aegypti) larvae over traps with no larvae or high density of heterospecific (Ae. aegypti) larvae. In the semi-field cage experiment, traps with low density of Ae. albopictus were not preferred more or less than any other trap, but in the open-field experiment they were preferred over traps without larvae.

Conclusions: We demonstrate, through open-field and semi-field cage experiments, that Ae. albopictus are more attracted to oviposition sites with larvae and that the combination of species and density of larvae influence attraction. This demonstrated preference could increase interspecific larval competition as Ae. albopictus actively seek containers with conspecific and heterospecific larvae. Any resulting competition with Ae. aegypti may favor one species over the other and alter the distribution or abundance of both. Because these species vary in vectorial capacity and insecticide resistance, effects of interspecific competition could ultimately impact arbovirus transmission rates and the success of vector control efforts.

Keywords: Aedes aegypti, Aedes albopictus, Interspecific competition, Oviposition choice, Mark-release-Recapture, Semi-field cage

\footnotetext{
*Correspondence: Ich27@cornell.edu

${ }^{1}$ Department of Entomology, Cornell University, Ithaca, NY, USA

Full list of author information is available at the end of the article
} 


\section{Background}

Aedes aegypti and Aedes albopictus mosquitoes are two highly successful invasive species that transmit the most important arboviruses impacting human health, including dengue, yellow fever, Zika and chikungunya. Aedes aegypti has spread throughout the global tropics, while Ae. albopictus has colonized every continent except Antarctica [1]. Aedes albopictus is currently invading Medellín, Colombia, where Ae. aegypti is well established, and both species have recently begun to coexist in some urban regions of Medellín [2]. Both Ae. albopictus and Ae. aegypti deposit their eggs above the water line in natural and artificial containers, thrive in urban/peri-urban environments, and are readily anthropophagic $[3,4]$. Their overlapping ecological niches result in high encounter rates; interestingly, the results of these interactions vary. In some cases, species overlap results in competitive replacement of $A e$. aegypti by Ae. albopictus [5, 6], while the reverse can also occur [7], and at times these two species stabilize and coexist [8].

The long-term outcome of co-occurrence on the distribution and abundance of each species has significant public health impacts. Aedes aegypti and Ae. albopictus differ in their feeding behavior [9] and their ability to transmit pathogens and parasites [10], meaning a shift in species abundance could alter the spread or intensity of arboviral disease. Aedes aegypti and Ae. albopictus also vary in insecticide resistance status [11], so efficient control strategies rely on understanding species distribution. Increased clarification of the ecological drivers behind competitive dynamics will enable more accurate predictions for the spread of these vectors, which will in turn allow for better-informed disease mitigation.

There are many hypotheses for why coexistence versus competitive exclusion may occur [12]. Larval competition is the most cited and commonly tested theory, and almost all studies agree that, when forced to share the same aquatic habitat, larval Ae. albopictus outcompete Ae. aegypti $[5,13]$. However, because interspecific encounters do not always result in competitive exclusion of Ae. aegypti [7, 8], other determinants must be at play.

One understudied factor in larval competition is the role of mosquito oviposition behavior. In order for larval competition to favor Ae. albopictus over Ae. aegypti, the two species must first deposit their eggs (oviposit) in the same containers. Mosquitoes do not choose oviposition sites at random; rather, they respond to multimodal cues to select preferred habitat [14-17]. If Ae. albopictus actively seek or avoid oviposition sites based on the presence or absence of Ae. aegypti or Ae. albopictus larvae, this would increase or decrease the degree of competition. However, experiments to date have mostly been laboratory-based $[18,19]$ or observational $[7,20]$. There is one published open-field-based study on how oviposition site preference can affect interspecific larval competition. This study collected naturally occurring eggs in containers with water used to previously rear varying levels of pre-existing conspecific and heterospecific larval density [21], and found evidence that these factors can drive Ae. albopictus oviposition site usage. However, the direction of this preference was unclear.

We conducted an experiment to test the hypothesis that Ae. albopictus oviposition site attraction in Medellín Medellín, Colombia, is driven by the presence, density, and species of either conspecific or heterospecific Ae. aegypti larvae in the aquatic habitat. While laboratory experiments control for environmental variability, they cannot always be extrapolated to the field, and while observational studies and natural collections report on true conditions, their design makes it difficult to pinpoint the ultimate cause of observed patterns. A combination of semi-field cage trials conducted under natural environmental conditions and open-field experiments with laboratory-reared cohorts of ovipositing females can maximize experimental control without losing real-world applicability. To this end, we first tested this hypothesis in the open-field using mark-release-recapture, and then replicated the experiment under semi-field cage conditions.

\section{Methods \\ General methods \\ Study location}

We conducted the semi-field cage experiment on the Universidad de Antioquia campus, 6 ${ }^{\circ} 16^{\prime} 4.97^{\prime \prime}$, - 75 347.91" and the open-field experiment in Barrio Santa Cruz, Medellín, Antioquia, Colombia, $6^{\circ} 17^{\prime} 44.42^{\prime \prime}$, - 75 33'11.76" (Fig. 1). Aedes aegypti has been established in both neighborhoods over a long period of time while Ae. albopictus was first detected through systematic city-wide ovitrap surveillance in Barrio Santa Cruz and in Barrio Universidad de Antioquia in 2011 (Secretaria de Salud Medellín, unpublished data). During the month-long semi-field cage study and the three-month open-field study, the average Medellín temperature was $21.6{ }^{\circ} \mathrm{C}$ and the average rainfall was $119 \mathrm{~mm}$. Santa Cruz is a dense urban residential community in the steep hills in the northeast of Medellín, with a population of 430 inhabitants per hectare. The government of Medellín categorizes $96.6 \%$ of the Santa Cruz population as socioeconomic status 2 and $3.4 \%$ as status 1 based on a ranking system of 1 to 6 , with 1 being the lowest [22]. Most homes in Santa Cruz are open to the outdoors, without screens. 


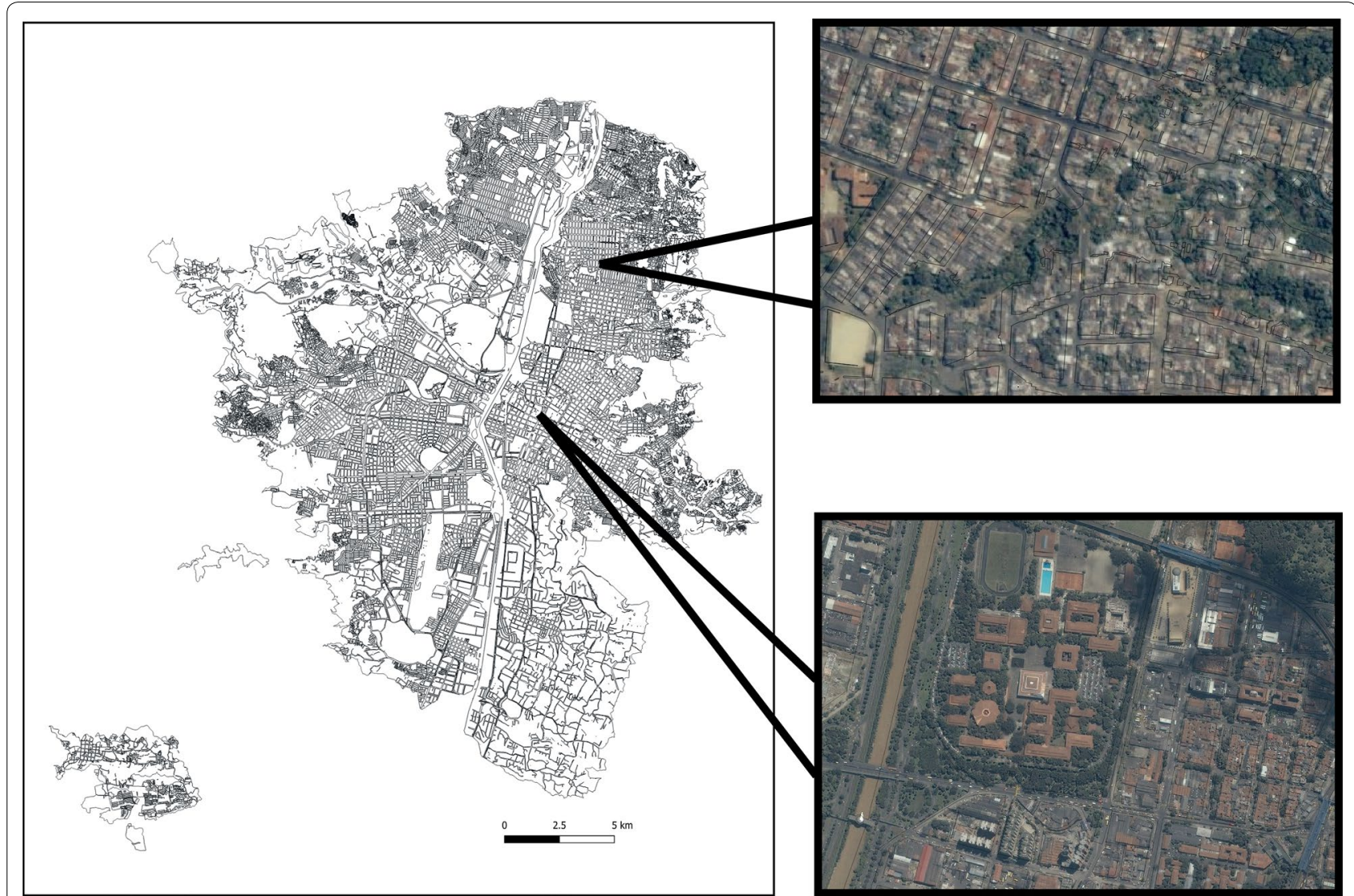

Fig. 1 Map of Medellín with close up satellite imagery showing the location of the field site (top) and semi-field cage site (bottom)

\section{Container survey and selection of release houses}

For this experiment to reflect accurate field conditions and to select release houses that were already positive for both species, we first surveyed naturally occurring larvae in Santa Cruz and determined the locations of oviposition sites. Although both can thrive indoors and outdoors, Ae. aegypti preferentially rest, feed, mate, and oviposit inside [23], while Ae. albopictus tend to be exophagic and exophilic [3]. Different cities have very different degrees of access to indoor/outdoor habitats and availability of indoor/outdoor oviposition sites, and this largely determines if larval habitat is located inside or outside the house, as in Moore et al. [24]. After obtaining permission from homeowners through door-to-door interviews, trained field personnel surveyed the indoor and outdoor areas of each house. Teams identified all water-holding containers suitable for larval development (defined by ability to hold at least $2 \mathrm{ml}$ of water and residents reported that they did not regularly clean and change the water) and recorded container location (indoor or outdoor) and presence or absence of mosquito larvae/pupae. Larval/pupal collection and species identification was as described in Shragai et al. [25].
We conducted the experimental release studies at three houses within Santa Cruz that were selected based on the following criteria: (i) full consent of the homeowner(s) was given; (ii) each contained outdoor space on the property; and (iii) each was found to have natural populations of both Ae. aegypti and Ae. albopictus, detected both as larvae and adults. Before each release, each home and all adjacent homes were thoroughly cleared of any potential oviposition sites.

\section{Mosquitoes and oviposition traps preparation}

Aedes albopictus used in the study were of low generation (F6-F8) adults from a colony established with fieldcollected larvae in Medellín, Antioquia, Colombia. Aedes aegypti used in the ovitraps were of similar low generation (F6-F8) Wolbachia-negative larvae from a colony established from Acacías, Meta, Colombia. These were known to be Wolbachia-negative because no Wolbachia releases have been conducted in Meta, Colombia. We did not release Ae. aegypti in this study.

All mosquitoes were hatched and reared as described previously (Alfonso-Parra et al. [26]. Released females 
were offered a blood meal from author Talya Shragai (TS) on days 5 and 6 post-eclosion, and all visibly engorged females were transferred to a separate cage provided with water, $10 \%$ sucrose ad libitum, and a daily human blood meal for 5 days until fully gravid.

One day post-hatch and four days pre-release, firstinstar larvae were placed in the experimental ovitraps. The ovitraps were filled with $3.7 \mathrm{l}$ of tap water at least 24 $\mathrm{h}$ prior to the addition of larvae to allow chlorine to dissipate. Experimental ovitraps contained two ground Hikari Gold Cichlid food pellets (mean weight of $3.56 \mathrm{~g} /$ pellet) (Hikari, Himeju, Japan) and one of two levels of larval density low density (20 larvae, density of 1 larva per 185 $\mathrm{ml}$ ) or high density (100 larvae, density of 1 larva per 37 $\mathrm{ml}$ ) of Ae. aegypti or Ae. albopictus. This nutrition level was chosen to be in surplus for both density treatments in order to equalize larval development rates and isolate preference based on signals received from healthy, nonstressed larvae.

\section{Trap design and experimental conditions}

Gravid females were recaptured using modified sticky ovitraps (Fig. 2c). Ovitraps were constructed from 11.4 1 black plastic buckets. The buckets were covered with mesh to ensure that no mosquitoes could escape if they eclosed in the trap and to prevent any mosquitoes from successfully laying eggs in the traps and potentially affecting semiochemicals in the oviposition water. A 1.9 l clear plastic food storage container with a $7.6 \mathrm{~cm}$ hole cut into the center bottom was fitted upside down on top of the bucket, and the sides of the plastic container were lined with clear plastic sheeting coated in Tangle Trap (Scotts Miracle-Gro, Marysville, OH). A 0.41 black plastic cup with the bottom removed was fitted into the hole in the clear plastic container. Each experimental block included four ovitraps representing each larval density-species combination and two control ovitraps that contained water and fish food but no larvae.

\section{Open-field experiment}

To test if Ae. albopictus attraction to oviposition sites is driven by the presence, density, and species of either conspecific or heterospecific Ae. aegypti larvae in an open field environment, we conducted a series of markrelease-recaptures using sticky ovitraps seeded with varying densities and species of larvae.

\section{Mark-release-recapture}

We marked mosquitoes on the day of release with DayGlo (DayGlo Color, Cleveland, OH, USA) fluorescent dust following the methods of Edman et al. [27], Harrington et al. [28], and others (Fig. 2). This method does not affect the fitness of the mosquitoes; most mosquitoes are marked for life (Harrington, unpublished data). We used a different color dust for each release site to quickly differentiate location of origin.

We conducted eight rounds of releases between 12 June and 8 August 2018. Mosquitoes were transferred to $473 \mathrm{ml}$ plastic cups for transportation to the release sites. Gravid females were released in the late afternoon (15:00-17:00 h), adjacent to ovitraps. Between 25 and 45 females were released in each of three houses in each round as described above.

Gravid females were recaptured using the sticky ovitraps described above, using the same experimental species and density conditions. Based on the results from the container survey, ovitraps were placed in the backyard of each release house in the location best representing natural Ae. albopictus larval habitat in urban residential Medellín. Ovitraps were checked every $24 \mathrm{~h}$ for four days starting $24 \mathrm{~h}$ after release. All captured mosquitoes were examined in the field to record species, sex, and presence/absence of fluorescent dust, and mosquitoes were then removed and discarded.

\section{Semi-field cage experiment}

To test if Ae. albopictus attraction to oviposition sites is driven by the presence, density, and species of either conspecific or heterospecific Ae. aegypti larvae under more highly controlled conditions, we replicated our open-field experiment in a semi-field cage.

\section{Semi-field cage}

The semi-field cage consisted of a $3.0 \times 4.5 \times 1.9 \mathrm{~m}$ $(\mathrm{w} \times \mathrm{l} \times \mathrm{h})$ tent cage (Fig. $2 \mathrm{a})$ made of white PVC coated polyester mesh, reinforced at angles and seams with white canvas tarp and supported by an aluminum frame. The cage was accessed using a front zipper door. The floor of the cage was made of white plastic for easy visualization of mosquitoes. The cage was placed under full tree cover to protect it from direct sunlight. The following items were placed inside the cage to provide refugia for mosquitoes (Fig. 2b, c): two potted plants (Beaucarnea sp., and Hibiscus sp.), four dry plastic buckets resting sideways, and a black cloth. Plants were watered as needed and leaves were misted daily to provide drinking water for released mosquitoes.

Ovitraps were placed in a circle around the center of the semi-field cage. The order of ovitraps was kept the same throughout the experiment, but the position was rotated one place clockwise between each replicate to identify any positional bias. 

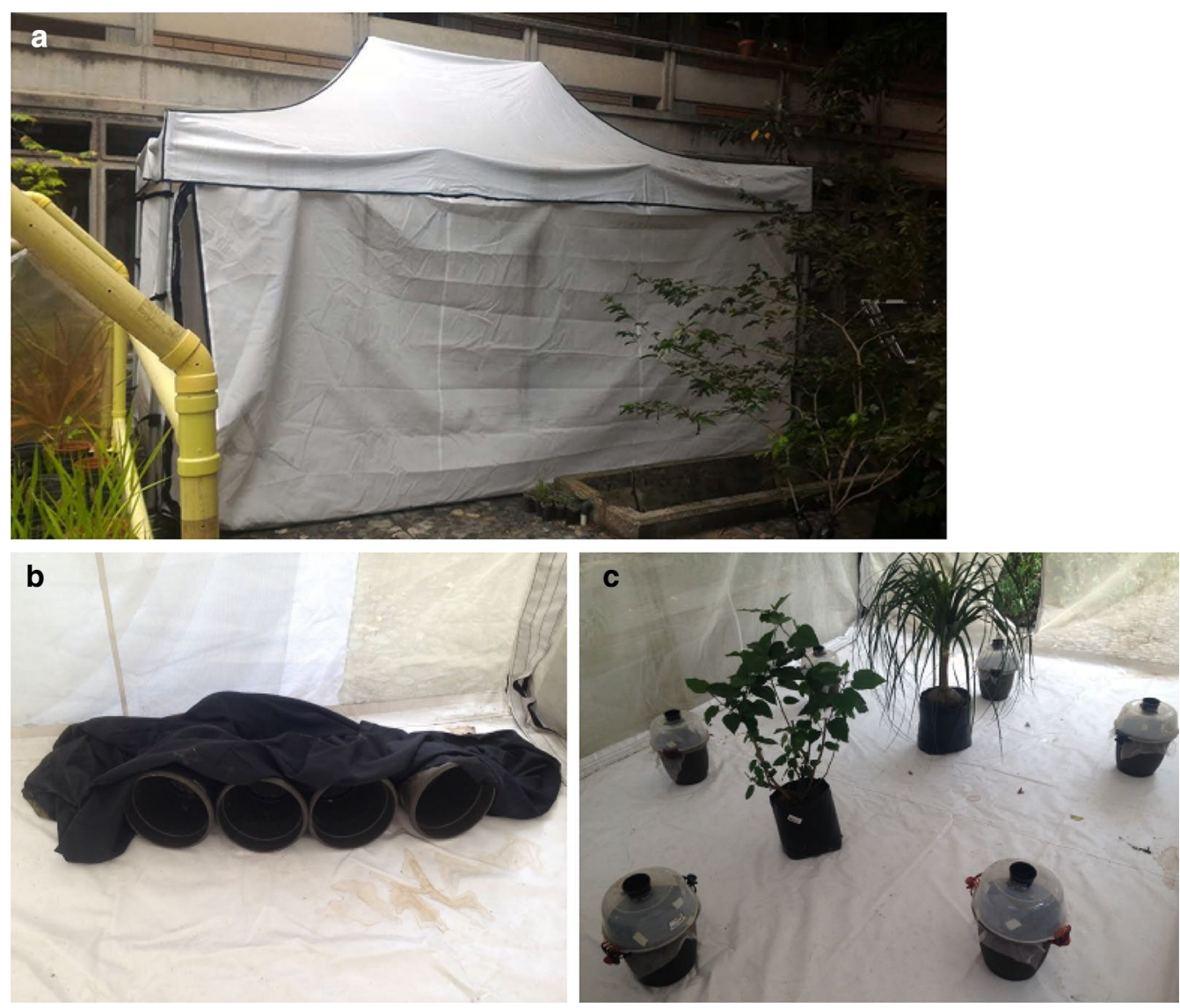

Fig. 2 Set-up of the semi-field cage. a Field cage exterior. b Field cage interior, showing close-up view of buckets with cloth. c Field cage interior, showing placement of ovitraps and resting sites: buckets with cloth and plants

\section{Release-recapture}

We conducted four different releases between 19 January and 19 February 2019. Females were released in the afternoon (13:00-16:00 h), in the center of the semi-field cage. Between 22 and 62 females were released in each replicate. Mosquitoes that did not voluntarily fly from their container when the lid was opened and the side tapped, were transported back to the laboratory and were not counted in the total number released. A point temperature reading both inside and outside the semi-field cage was taken when the mosquitoes were released and on each recapture day to ensure that temperatures inside the cage accurately reflected environmental conditions.

Ovitraps were checked every $24 \mathrm{~h}$ for two days starting $24 \mathrm{~h}$ after release. All captured mosquitoes were removed and discarded. Any remaining uncaptured mosquitoes were killed with an electric racket at the end of each replicate (Black Flag, Madison, WI, USA).

\section{Data analysis}

The open-field data and semi-field cage data were analyzed individually using separate models. For both, we used a generalized linear mixed model using Poisson distributions to analyze the effects of ovitrap treatment on Ae. albopictus recapture. Date of release and ovitrap ID were included as random factors and for the open-field experiment, house of release was included as a blocking factor. The number of Ae. albopictus recaptured over the experiment was used as the dependent variable, and four forms of fixed effects structures were used: larvae/no larvae, species, density, and each of the five species-density combinations. For the response variable, the number recaptured for all days in each trap of each release replicate was totaled. Further pairwise analysis was conducted by calculating the estimated marginal means and performing all pairwise comparisons. All analyses were conducted in R ( $\mathrm{R}$ Core Team, Version 3.5.2), using the lme4 and emmeans packages [29-31].

Although we collected wild, non-marked Ae. albopictus in the open-field study, we did not include an analysis of 
Table 1 Container survey results. Containers and houses negative and positive for Ae. aegypti and Ae. albopictus. Results are divided into containers and houses with containers located indoors and outdoors

\begin{tabular}{lll}
\hline & $\begin{array}{l}\text { Containers } \\
\text { surveyed }\end{array}$ & $\begin{array}{l}\text { Houses } \\
\text { surveyed }\end{array}$ \\
\hline $\begin{array}{lll}\text { Indoors } \\
\text { Positive, Ae. aegypti }\end{array}$ & 8 & 2 \\
Positive, Ae. albopictus & 0 & 0 \\
Positive, Ae. aegypti and Ae. albopictus & 0 & 0 \\
Negative & 19 & 370 \\
Total & 27 & 372 \\
Outdoors & & \\
Positive, Ae. aegypti & 29 & 28 \\
Positive, Ae. albopictus & 4 & 2 \\
Positive, Ae. aegypti and Ae. albopictus & 10 & 4 \\
Negative & 233 & 338 \\
Total & 276 & 372 \\
Total & 303 & 372 \\
\hline
\end{tabular}

these mosquitoes because the sample size (total $n=35$ ) was too low to detect statistical differences.

\section{Results}

\section{Open-field experiment Container survey}

Only $9.68 \%$ of the houses surveyed had at least one positive container (Table 1). Overall, $83.17 \%$ of 305 containers surveyed were negative and $16.83 \%$ were positive. Of the positive containers, $72.55 \%$ were positive for Ae. aegypti alone, $19.61 \%$ were positive for Ae. aegypti and Ae. albopictus, and $7.84 \%$ were positive for Ae. albopictus alone. Almost all containers identified in the survey, including those both positive and negative for larvae, were located indoors.

\section{Mark-release-recapture}

The overall recapture rate was $12.85 \%$ and the total number recaptured was 115 , which are numbers consistent with or higher than other studies using similar trapping methods $[29,30]$. The number of marked Ae. albopictus recaptured in oviposition containers was affected by the presence of larvae in the ovitraps with significantly more Ae. albopictus recaptured in traps with larvae of either species than in traps with no larvae $(P<0.0001$, $Z$-ratio $=-3.999, S E=0.357)$. Significantly more $A e$. albopictus were recaptured in traps with Ae. albopictus larvae at high densities $(P=0.0006, Z$-ratio $=-4.653$, $S E=0.0806)$ and in containers with Ae. aegypti larvae at low density $(P=0.0014, Z$-ratio $=-3.801, S E=0.0875)$ than in the no larvae control traps (Fig. 3). The number

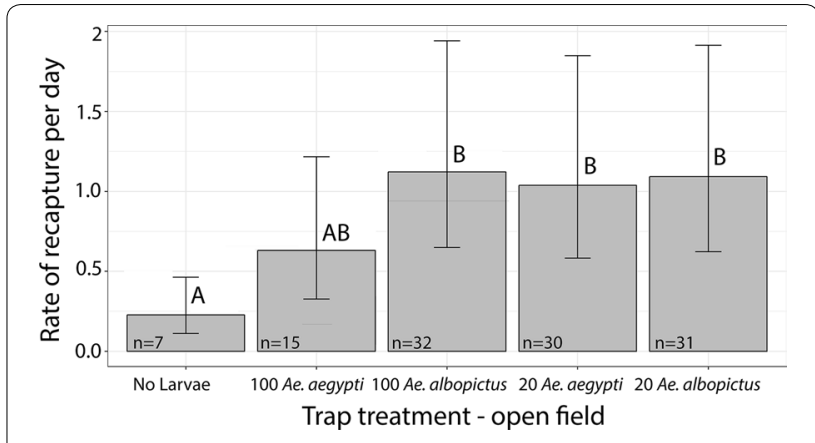

Fig. 3 Results of the open-field experiment showing marked-released-recaptured Ae. albopictus. Mean recapture rates per day $\pm 95 \%$ confidence intervals are reverse transformed from the estimated marginal means of our Poisson distributed generalized mixed model. Significantly different treatments are indicated by letters. More Ae. albopictus were recaptured in traps with $100 \mathrm{Ae}$. albopictus, 20 Ae. aegypti and 20 Ae. albopictus than in traps with no larvae $(P=0.0006, S E=0.0806 ; P=0.0014, S E=0.0875 ; P=0.0008$ $S E=0.082)$. The number of Ae. albopictus recaptured in traps with 100 Ae. aegypti was not significantly different than traps with any species/ density combination or the control traps

of Ae. albopictus recaptured in traps with high density Ae. aegypti was not significantly different than traps with any species/density combination (low density Ae. aegypti: $P=0.686, Z$-ratio $=-0.169, S E=0.608$; low density Ae. albopictus: $P=0.594, Z$-ratio $=-2.323, S E=0.578)$ nor than the control traps $(P=0.156, Z$-ratio $=-1.816$, $S E=0.082$ ) (Fig. 3). The number recaptured in ovitraps with low density Ae. albopictus was significantly higher than in the traps with no mosquito larvae $(P=0.0008$, $Z$-ratio $=-4.204, S E=0.082$ ) (Fig. 3). All other variables had non-significant effects.

\section{Semi-field cage experiment}

We recaptured a total of 117 females, for a recapture rate of $78.52 \%$. The number of Ae. albopictus recaptured in oviposition containers was affected by the presence of larvae in the ovitraps $(P=0.0260, Z$-ratio $=-2.072$, $S E=0.403)$. Just as in the open-field experiment, significantly more Ae. albopictus were recaptured in containers with Ae. albopictus at high densities $(P=0.0349$, $Z$-ratio $=-2.855, S E=0.127)$ and in containers with Ae. aegypti larvae at low densities $(P=0.0161$, $Z$-ratio $=-3.108, S E=0.115)$ than in the control traps with no larvae (Fig. 4). Again, the number of $A e$. albopictus recaptured in traps with high density of $A e$. aegypti was not significantly different than in traps with any species/density combination (low density of $A e$. aegypti: $P=0.186, Z$-ratio $=-2.183, S E=0.168$; low density of Ae. albopictus: $P=0.982, Z$-ratio $=-0.552$, $S E=0.384$, high density of Ae. albopictus: $P=0.282$, $Z$-ratio $=-1.968, S E=0.187$ ) or than in the control traps 


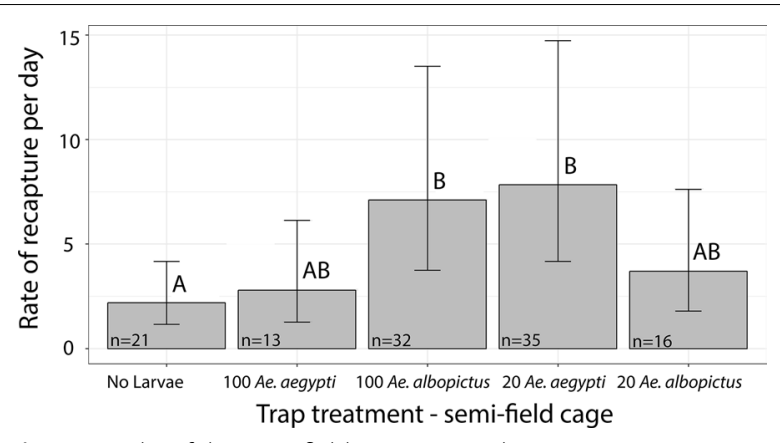

Fig. 4 Results of the semi-field experiment showing released-recaptured Ae. albopictus. Mean recapture rates per day \pm 95\% confidence intervals are reverse transformed from the estimated marginal means of our Poisson distributed generalized mixed model. Significantly different treatments are indicated by letters. More Ae. albopictus were recaptured in traps with $100 \mathrm{Ae}$. albopictus and 20 Ae. aegypti than in traps with no larvae $(P=0.0349, S E=0.127$; $P=0.0161, S E=0.115)$. The number of Ae. albopictus recaptured in traps with $100 \mathrm{Ae}$. aegypti and $20 \mathrm{Ae}$. albopictus was not significantly different than in traps with any species/density combination or the control traps

$(P=0.986, Z$-ratio $=-0.517, S E=0.365)$ (Fig. 4). In this experiment, the number recaptured in the trap with low density of Ae. albopictus was also not significantly different than in the control $(P=0.775, Z$-ratio $=-1.158$, $S E=0.267$ ) or the preferred traps (high density of $A e$. albopictus: $P=0.598, Z$-ratio $=1.446, S E=0.871$; low density of Ae. aegypti: $P=0.453, Z$-ratio $=1.670$, $S E=0.954$ ) (Fig. 4). All other variables had non-significant effects.

\section{Discussion}

This study addresses female Ae. albopictus oviposition choices that may lead to larval competition with Ae. aegypti. We tested attraction to ovitraps containing either conspecific larvae or heterospecific Ae. aegypti larvae at two levels of density in both the open-field and a semi-field cage. We showed that Ae. albopictus preferentially seek oviposition sites that contain larvae over those that do not, and that the combination of species and density of larvae in the container influenced oviposition site choice; those with high densities of conspecific larvae or low densities of heterospecific larvae were most attractive. Our results support previous laboratory studies and show that this behavior is relevant in the field.

Our semi-field cage and open-field experiments demonstrated that Ae. albopictus preferentially seek oviposition sites that contain larvae over those that do not. These results are corroborated by previous laboratory studies demonstrating that Ae. albopictus preferentially oviposit in sites that already contain larvae [18, 32-34]. This attraction is thought to be because the presence of conspecific larvae can indicate high-quality habitat; in $A e$. aegypti it has been hypothesized to signal adequate food and infrequent desiccation [35]. We chose to use a consistent initial amount of larval nutrition (mean weight of 3.56 g per trap) rather than a set amount per larvae in the trap, which means that over the course of each replicate the nutrition was differentially depleted by larvae in each experimental treatment. However, if oviposition attraction was solely based on nutrient levels, we would expect the treatments with more larvae to be less attractive. We did not see this effect, further supporting our conclusion that larvae produce cues independent of nutrient levels that influence gravid female oviposition site selection.

We recaptured more gravid Ae. albopictus females in ovitraps with 100 Ae. albopictus than in traps with no larvae in both the open-field experiment and the semifield cage experiment. Mosquitoes should seek oviposition sites that optimize offspring fitness, which is a balance between ensuring sufficient resources and minimizing detrimental competition. Although conspecific larvae can signal sight productivity, very high larval density lengthens development time and decreases adult mosquito size, thereby reducing fitness [34]. Therefore, attraction to conspecific larvae has been theorized to be hump-shaped density-dependent, with attraction increasing with greater numbers of larvae until a critical maximum, after which density is too high, and attraction declines. In an open-field study of Ae. albopictus oviposition site preference based on just conspecific larvae, preference increases with increasing numbers of larvae in the container until 130 larvae/0.37 I water, and decreased if larval density was greater [36]. This result is consistent with our findings that 100 conspecific larvae/3.7 l, a density level well before the critical maximum, was more attractive to Ae. albopictus adults than containers with no larvae.

More Ae. albopictus were recaptured in traps with 20 Ae. aegypti than with no larvae, but Ae. albopictus showed no preference for containers with $100 \mathrm{Ae}$. aegypti compared to larvae-free containers. There is an overlap in preferred larval environment for Ae. aegypti and Ae. albopictus, so the presence of heterospecific larvae may still be used to detect high-quality habitat, as is the case in other mosquito systems [37-39]. We suggest that, while heterospecific larvae may be attractive, the density tolerance threshold might be lower and dependent on existing resource levels. In our study, it may be that the combination of low numbers of heterospecific larvae with high resource surplus was attractive, but they had an intermediary preference towards containers with a greater number of Ae. aegypti larvae and a lesser surplus of nutrients. However, the results from previous studies are sparse and inconsistent. One laboratory study 
showed that Ae. albopictus prefers to oviposit in water used to rear Ae. aegypti [18]. A field study found that Ae. albopictus preferred water that had never contained larvae over water used to rear Ae. aegypti under nonstressful nutrient conditions [21]. Another laboratory study [19] found no attraction to "low" and "medium" (10 or 100 larvae/60 $\mathrm{ml}$ water) densities of Ae aegypti larvae and strong preferential oviposition to "high" (500 larvae/60 ml water) densities compared to an ovicup with no larvae, but medium and high densities in this paper are far above those observed in the field.

The stark contrast between these three studies may be due to their methodological differences or to genetic variation between strains used. Each of these protocols varied in the larval densities tested, the use of water containing larvae versus strained larval rearing water, and if they were conducted in the laboratory versus in the field. It is also of note that all the previous studies used sieved water previously used for rearing larvae or placed washed larvae in clean water, which also contrasts with our methodology. Alternatively, the different results could be due to genetic variation between the mosquito strains. These species can undergo rapid local adaptation, and strain variation has been shown to be responsible for behavioral differences such as escape response to pesticides [40, 41], mating behavior [42, 43], and larval alarm reactions [44]. Furthermore, the interspecific competitive ability of $A e$. aegypti against Ae. albopictus at the adult stage has been shown to vary by location. Strains of Ae. aegypti with previous exposure to Ae. albopictus are less susceptible to satyrization than those with minimal interspecific exposure [45]. It may be that susceptibility to larval competition varies by strain as well, which could consequently impact oviposition choice by strain. Future research could test a range of mosquito strains, and vary larval densities and resource levels to pinpoint their respective effects on oviposition preference.

It is unclear why there was a significant preference for traps containing $20 \mathrm{Ae}$. albopictus over the traps with no larvae in the open-field experiment, but no significant preference for or against this treatment in the semi-field cage experiment. If our results were to support the theory that attraction to conspecific larvae increases as the density increases until 130 larvae/0.37 l, we would expect our 100 Ae. albopictus treatment to be most attractive, our $20 \mathrm{Ae}$. albopictus treatment to be intermediately attractive, and our control to be least attractive. This was, in fact, the case in the semi-field cage, but not in the openfield. This may be because other environmental cues were present in the open-field setting, or because of an undetected effect of the bucket used.

The highly significant preference for containers with larvae with our sample size indicates that this preference is strong, and the similarity of the open-field and semifield cage findings further corroborate our conclusions. Our results contradict one previous field study which found an idiosyncratic preference of Ae. albopictus for ovicups with con- and hetero-specific larvae [21]. This discrepancy may be due to methodological variation. While we used 5-day-old larvae in the ovitraps, they raised mosquitoes to eclosion and used sieved rearing water. These approaches reflect equally appropriate but different field conditions. Furthermore, their experimental containers were open-air, and they used total eggs laid as their response. Attraction to semiochemicals by mosquitoes often occurs over long distances, but any gustatory or tactile oviposition cues require contact with the water $[46,47]$. Our ovitrap design did not permit females to touch water, and so our results only reflect preference for cues detected at a range. Further experimentation should compare these cues and their effect on active oviposition.

Aedes aegypti were not released in this study. The World Mosquito Programme is currently releasing Wolbachia-positive Ae. aegypti in Medellín and maintains a proprietary right on these mosquitoes. In order to conduct field trials with Ae. aegypti, researchers must use Medellín-origin Ae. aegypti that are confirmed negative for Wolbachia, and we were unable to create a compliant laboratory colony within our time frame.

\section{Conclusions}

The invasion of Medellín, Colombia by Ae. albopictus is a recent and ongoing phenomenon. Any resulting competition with Ae. aegypti could preferentially favor one species over the other and alter the distribution or abundance of both species. This in turn could have implications on the spread of arboviral disease. Medellín is still predominantly inhabited by Ae. aegypti, and larval populations are low enough that ovipositing females can select between containers with or without other larvae. Because oviposition site-seeking Ae. albopictus actively are attracted to those with both conspecific and heterospecific mosquitoes, larval competition should be exacerbated as Ae. albopictus continues to invade. Further studies are needed to understand the impact of interspecific larval competition on the fitness of both species and to understand in more detail how ecological context modifies the outcome of oviposition behavior and interspecific competition.

\section{Abbreviation}

SE: standard error. 


\section{Acknowledgements}

We are grateful for the support of the Secretaria de Salud, Alcadia de Medellín, Colombia, without whom this project would have been impossible. We are especially grateful to Dr. Guillermo L. Rua Uribe, Juliana Perez and Marcela Quimbayo Forero for their advice and support. We appreciate the statistical consulting of Erika Mudrak, RutaN Medellín for technical support, and the field assistance of Paulina Gutierrez Arbelaez, Edison Trujilo, and Carolina Bedoya.

\section{Authors' contributions}

TS conceived and designed the experiment, conducted all field and laboratory work, performed all data analysis, and was the major writer of the manuscript. CA and FA were the major coordinators of experimental logistics. LH was a major contributor to the experimental design and writing the manuscript. All authors read and approved the final manuscript.

\section{Funding}

This project was funded in part from the Cornell Graduate School Research Travel Fund, in part from the Cornell Department of Entomology Rawlins Fund and Griswold Fund, in part by COLCIENCIAS, Universidad de Antioquia and the Max Planck Society cooperation grant 566-1 (2014) (to FWA), in part by COLCIENCIAS grant CD 325671250804 (CT 409-2016) (to CAP), and in part by the National Institute of Food and Agriculture (NIFA), U.S. Department of Agriculture, Multistate Hatch Project under 2017-18-225.

\section{Availability of data and materials}

The datasets used and/or analyzed during the current study are available from the corresponding author on reasonable request.

\section{Ethics approval and consent to participate}

All experimental procedures were approved by the Secretaría de Salud, Alcaldía de Medellín (Medellín Secretary of Health) and reviewed and determined exempt by Cornell University's Institutional Review Board (IRB). After obtaining approvals, we explained the project and obtained consent from residents in the study community. Mark-release-recapture studies for Aedes mosquitoes generally remove more mosquitoes from the environment than are released, decreasing the overall risk of disease transmission [48]. However, numerous safety measures were taken to further minimize human risk. We explained and provided written documentation of all experimental procedures to participating residents and provided information about how to decrease Aedes larval habitat, minimize biting risk, and recognize the symptoms of dengue, Zika and chikungunya. Residents could end their participation in the project at any time. In addition, we committed to halting all experiments if there was a reported arboviral case in the neighborhood. Residents were further provided with mosquito repellent (OFF 30\%, SC Johnson, Racine, WI) and an electric insect racket (Black Flag, Madison, WI). All participating residences were thoroughly checked and cleared of any water-holding container that could serve as larval habitat.

\section{Consent for publication}

Not applicable.

\section{Competing interests}

The authors declare that they have no competing interests.

\section{Author details}

${ }^{1}$ Department of Entomology, Cornell University, Ithaca, NY, USA. ${ }^{2}$ Instituto Colombiano de Medicina Tropical, Universidad CES, Sabaneta 055450 Antioquia, Colombia. ${ }^{3}$ Max Planck Tandem Group in Mosquito Reproductive Biology, Universidad de Antioquia, Medellín 050010, Antioquia, Colombia.

Received: 7 May 2019 Accepted: 7 September 2019 Published online: 18 September 2019

\section{References}

1. Kraemer MU, Sinka ME, Duda KA, Mylne AQ, Shearer FM, Barker CM, et al. The global distribution of the arbovirus vectors Aedes aegypti and Ae. albopictus. Elife. 2015;4:e08347.
2. Rúa-Uribe G, Suárez-Acosta C, Londoño V, Sánchez J, Rojo R, Bello-Novoa B. Detección de Aedes albopictus (Skuse) (Diptera: Culicidae) en la ciudad de Medellín, Colombia. Biomédica. 2011;31(Supl. 3):243-4.

3. Hawley WA. The biology of Aedes albopictus. J Am Mosq Control. 1988;1(Suppl.):1-39.

4. Ponlawat A, Harrington LC. Blood feeding patterns of Aedes aegypti and Aedes albopictus in Thailand. J Med Entomol. 2005;42:844-9.

5. Juliano SA. Species introduction and replacement among mosquitoes: interspecific resource competition or apparent competition? Ecology. 1998;79:255-68.

6. Lounibos LP. Invasions by insect vectors of human disease. Annu Rev Entomol. 2002;47:233-66

7. Chan KL. Aedes aegypti (L.) and Aedes albopictus (Skuse) in Singapore-City. 4. Competition between species. Bull World Health Organ. 1971;44:651-7.

8. Juliano SA, Lounibos LP, O'Meara GF. A field test for competitive effects of Aedes albopictus on A. aegypti in South Florida: differences between sites of coexistence and exclusion? Oecologia. 2004;139:583-93.

9. Thavara U, Tawatsin A, Chansang C, Kong-ngamsuk W, Paosriwong S, Boon-Long J, et al. Larval occurrence, oviposition behavior and biting activity of potential mosquito vectors of dengue on Samui Island, Thailand. J Vector Ecol. 2001;26:172-80.

10. Lounibos LP, Kramer LD. Invasiveness of Aedes aegypti and Aedes albopictus and vectorial capacity for chikungunya virus. J Infect Dis. 2016;214(Suppl. 5):S453-8.

11. Ponlawat A, Scott JG, Harrington LC. Insecticide susceptibility of Aedes aegypti and Aedes albopictus across Thailand. J Med Entomol. 2005;42:821-5.

12. Lounibos LP. Competitive displacement and reduction. J Am Mosq Control. 2007;23:276-82.

13. Barrera R. Competition and resistance to starvation in larvae of containerinhabiting Aedes mosquitoes. Ecol Entomol. 1996;21(2):117-27.

14. Harrington LC, Ponlawat A, Edman JD, Scott TW, Vermeylen F. Influence of container size, location, and time of day on oviposition patterns of the dengue vector, Aedes aegypti, in Thailand. Vector Borne Zoonotic Dis. 2008:8:415-23.

15. Yee DA, Kneitel JM, Juliano SA. Environmental correlates of abundances of mosquito species and stages in discarded vehicle tires. J Med Entomol. 2010:47:53-62.

16. Davis TJ, Kaufman PE, Hogsette JA, Kline DL. The effects of larval habitat quality on Aedes albopictus skip oviposition. J Am Mosq Control. 2015;31:321-8.

17. Wasserberg G, White L, Bullard A, King J, Maxwell R. Oviposition site selection in Aedes albopictus (Diptera: Culicidae): are the effects of predation risk and food level independent? J Med Entomol. 2013;50:1159-64.

18. Allan SA, Kline DL. Larval rearing water and preexisting eggs influence oviposition by Aedes aegypti and Ae. albopictus (Diptera: Culicidae). J Med Entomol. 1998;35:943-7.

19. Gonzalez PV, Gonzalez Audino PA, Masuh HM. Oviposition behavior in Aedes aegypti and Aedes albopictus (Diptera: Culicidae) in response to the presence of heterospecific and conspecific larvae. J Med Entomol. 2016:53:268-72.

20. Braks MA, Honorio NA, Lourencqo-De-Oliveira R, Juliano SA, Lounibos LP. Convergent habitat segregation of Aedes aegypti and Aedes albopictus (Diptera: Culicidae) in southeastern Brazil and Florida. J Med Entomol. 2003:40:785-94.

21. Fader JE, Juliano SA. Oviposition habitat selection by container-dwelling mosquitoes: responses to cues of larval and detritus abundances in the field. Ecol Entomol. 2014;39:245-52.

22. Medellín A. Perfil total Medellín. In: Encuesta Calidad de Vida, Medellín. La Alcaldia de Medellín Departamento Administrativo de Planeación; 2011. https://www.Medellín.gov.co/irj/go/km/docs/wpccontent/Sites/ Subportal\%20del\%20Ciudadano/Planeaci\%C3\%B3n\%20Municipal/Secciones/Publicaciones/Documentos/Encuesta\%20Calidad\%20de\%20Vida/ ECV2011/Perfil\%20Total\%20Medellín\%20Encuesta\%20Calidad\%20de\%20 Vida\%202011.pdf. Accessed 30 July 2019.

23. Christophers SR. Aedes aegypti: the yellow fever mosquito. London: CUP Archive; 1960.

24. Moore CG, Cline BL, Ruiz-Tiben E, Lee D, Romney-Joseph H, Rivera-Correa E. Aedes aegypti in Puerto Rico: environmental determinants of larval abundance and relation to dengue virus transmission. Am J Trop Med Hyg. 1978;27:1225-31. 
25. Shragai T, Harrington LC. Aedes albopictus (Diptera: Culicidae) on an invasive edge: abundance, spatial distribution, and habitat usage of larvae and pupae across urban and socioeconomic environmental gradients. J Med Entomol. 2019;56:472-82.

26. Alfonso-Parra C, Ahmed-Braimah YH, Degner EC, Avila FW, Villarreal SM, Pleiss JA, et al. Mating-induced transcriptome changes in the reproductive tract of female Aedes aegypti. PLoS Neglect Trop Dis. 2016;10:e0004451.

27. Edman JD, Scott TW, Costero A, Morrison AC, Harrington LC, Clark GG Aedes aegypti (Diptera: Culicidae) movement influenced by availability of oviposition sites. J Med Entomol. 1998;35:578-83.

28. Harrington LC, Scott TW, Lerdthusnee K, Coleman RC, Costero A, Clark GG, et al. Dispersal of the dengue vector Aedes aegypti within and between rural communities. Am J Trop Med Hyg. 2005;72:209-20.

29. R Core Team. R: a language and environment for statistical computing. Vienna: R Foundation for Statistical Computing;: 2017. https://www.Rproject.org/. Accessed 15 June 2019.

30. Marini F, Caputo B, Pombi M, Tarsitani G, Della Torre A. Study of Aedes albopictus dispersal in Rome, Italy, using sticky traps in mark-releaserecapture experiments. Med Vet Entomol. 2010;24:361-8.

31. Maciel-De-Freitas R, Codeco CT, Lourenco-De-Oliveira R. Daily survival rates and dispersal of Aedes aegypti females in Rio de Janeiro, Brazil. Am J Trop Med Hyg. 2007;76:659-65.

32. Gubler DJ. Studies on the comparative oviposition behavior of Aedes (Stegomyia) albopictus and Aedes (Stegomyia) polynesiensis Marks. J Med Entomol. 1971;8:675-82.

33. Yap HH, Lee CY, Chong NL, Foo AE, Lim MP. Oviposition site preference of Aedes albopictus in the laboratory. J Am Mosq Control Assoc. 1995;11:128-32.

34. Yoshioka M, Couret J, Kim F, McMillan J, Burkot TR, Dotson EM, et al. Diet and density-dependent competition affect larval performance and oviposition site selection in the mosquito species Aedes albopictus (Diptera: Culicidae). Parasites Vectors. 2012;5(1):225.

35. Wong J, Stoddard ST, Astete H, Morrison AC, Scott TW. Oviposition site selection by the dengue vector Aedes aegypti and its implications for dengue control. PLoS Negl Trop Dis. 2011;5:e1015.

36. Wasserberg G, Bailes N, Davis C, Yeoman K. Hump-shaped densitydependent regulation of mosquito oviposition site-selection by conspecific immature stages: theory, field test with Aedes albopictus, and a meta-analysis. PLoS ONE. 2014;9(3):e92658.

37. Zahiri N, Rau ME, Lewis DJ. Oviposition responses of Aedes aegypti and Ae. atropalpus (Diptera: Culicidae) females to waters from conspecific and heterospecific normal larvae and from larvae infected with Plagiorchis elegans (Trematoda: Plagiorchiidae). J Med Entomol. 1997;34:565-8.
38. Maire A, Langis R. Oviposition responses of Aedes (Ochlerotatus) communis (Diptera: Culicidae) to larval holding water. J Med Entomol. 1985:22:111-2.

39. McDaniel IN, Bentley MD, Lee H-P, Yatagai M. Studies of Aedes triseriatus (Diptera: Culicidae) oviposition attractants. Evidence for attractant production by kaolin-treated larvae. Can Entomol. 1979;111:143-7.

40. Kongmee M, Prabaripai A, Akratanakul P, Bangs MJ, Chareonviriyaphap T. Behavioral responses of Aedes aegypti (Diptera: Culicidae) exposed to deltamethrin and possible implications for disease control. J Med Entomol. 2004:41:1055-63.

41. Thanispong K, Achee NL, Bangs MJ, Grieco JP, Suwonkerd W, Prabaripai A, Chareonviriyaphap T. Irritancy and repellency behavioral responses of three strains of Aedes aegypti exposed to DDT and a-cypermethrin. J Med Entomol. 2009;46:1407-14.

42. Black WC IV, Rai KS, Turco BJ, Arroyo DC. Laboratory study of competition between United States strains of Aedes albopictus and Aedes aegypti (Diptera: Culicidae). J Med Entomol. 1989;26:260-71.

43. Ponlawat A, Harrington LC. Factors associated with male mating success of the dengue vector mosquito, Aedes aegypti. Am J Trop Med Hyg. 2009;80:395-400

44. Duhrkopf RE, Benny H. Differences in the larval alarm reaction in populations of Aedes aegypti and Aedes albopictus. J Am Mosq Control. 1990;6:411-4.

45. Bargielowski IE, Lounibos LP, Carrasquilla MC. Evolution of resistance to satyrization through reproductive character displacement in populations of invasive dengue vectors. Proc Natl Acad Sci USA. 2013;110:2888-92.

46. Bentley MD, Day JF. Chemical ecology and behavioral aspects of mosquito oviposition. Annu Rev Entomol. 1989;34:401-21.

47. Matthews BJ, Younger MA, Vosshall LB. The ion channel ppk301 controls freshwater egg-laying in the mosquito Aedes aegypti. Elife. 2019;8:e43963.

48. Benedict MQ, Charlwood JD, Harrington LC, Lounibos LP, Reisen WK, Tabachnick WJ. Guidance for evaluating the safety of experimental releases of mosquitoes, emphasizing mark-release-recapture techniques. Vector Borne Zoonotic Dis. 2018;18:39-48.

\section{Publisher's Note}

Springer Nature remains neutral with regard to jurisdictional claims in published maps and institutional affiliations.
Ready to submit your research? Choose BMC and benefit from:

- fast, convenient online submission

- thorough peer review by experienced researchers in your field

- rapid publication on acceptance

- support for research data, including large and complex data types

- gold Open Access which fosters wider collaboration and increased citations

- maximum visibility for your research: over $100 \mathrm{M}$ website views per year

At $\mathrm{BMC}$, research is always in progress.

Learn more biomedcentral.com/submissions 